\title{
Diversidad florística asociada a las lagunas andinas Pomacocha y Habascocha, Junín, Perú
}

\section{Floristic diversity associated to andean Pomacocha and Habascocha Lakes, Junín, Peru}

\author{
Mercedes Flores, José Alegría y Arturo Granda*
}

Presentado: 23/04/2004

Aceptado: 20/05/2005

\section{Resumen}

Se realizó un estudio de la diversidad florística de los alrededores de las lagunas Pomacocha y Habascocha (4350 - $4550 \mathrm{~m}$ de altitud, $11^{\circ} 45^{\prime}-11^{\circ} 48^{\prime} \mathrm{S}$ y $75^{\circ} 12^{\prime}-75^{\circ} 15^{\prime} \mathrm{O}$ ), Provincia de Concepción, Junín, Perú. Se han registrado 29 familias, 64 géneros y 100 especies. Poaceae fue la familia con mayor diversidad específica (25\%), seguida por Asteraceae (24\%) y Gentianaceae $(6 \%)$. Se hace una comparación de la composición florística de la puna de Concepción con la de otras localidades de la puna del Perú.

Palabras clave: Diversidad, florística, humedales, Andes, Perú.

\section{Abstract}

A study of the floristic diversity of the surroundings of Lakes Pomacocha and Habascocha (4350 $4550 \mathrm{~m}, 11^{\circ} 45^{\prime}-11^{\circ} 48^{\prime} \mathrm{S}$ and $75^{\circ} 12^{\prime}-75^{\circ} 15^{\prime} \mathrm{W}$ ), Province of Concepción, Junín, Peru, reported a total of 29 families, 64 genera, and 100 species. Poaceae was the most diverse family $(25 \%)$, followed by Asteraceae (24\%) and Gentianaceae (6\%). The floristic composition of the Puna of Concepción was compared to those of other localities of the Peruvian Puna.

Keywords: Diversity, floristics, wetlands, Andes, Peru.

\section{Introducción}

La flora que se desarrolla en los alrededores de las lagunas altoandinas es quizás una de las menos conocidas por su difícil acceso y por las condiciones extremas del clima que dificultan su estudio; no obstante, contribuciones recientes (Tovar, 1973, 1990; Tapia \& Flores, 1984; Smith, 1988; Young \& León, 1990; Young \& Cano, 1994) referidas a esta vegetación resultan de suma importancia.

Las plantas de los alrededores de las lagunas muestran adaptaciones morfoanatómicas a las peculiares condiciones medioambientales de la puna (Cabrera, 1968) que dan lugar a especies graminiformes, pulvinadas, enanas, acaules y postradas, así como el desarrollo de individuos en densas asociaciones. Tales formas de vida han sido descritas con algún detalle, entre otros autores, por Weberbauer (1945), Cabrera (1957), Luteyn (1996) y Sánchez (1996).

\footnotetext{
* Herbario del Departamento de Biología (MOL), Facultad de Ciencias. Universidad Nacional Agraria La Molina. Aptdo. 456, Lima, Perú
}

Los bofedales u oconales son formaciones altoandinas que generalmente ocupan terrenos inmediatos a lagunas o aguas de corriente lenta, en ellas el suelo está empapado en agua, completamente saturado, en estas vegas o sitios semipantanosos se desarrolla una vegetación higrófila siempre verde (Gómez, 1966) donde Distichia muscoides es el elemento dominante. Por su naturaleza, son lugares de pastoreo y éste se intensifica en la época de sequía (junio-octubre); por esta razón, muchas especies de plantas vulnerables tienden a desaparecer.

En los últimos 20 años, además se ha intensificado la extracción de la turbera de Distichia, para su utilización como combustible y fundamentalmente en la preparación de tierra vegetal en los viveros de Lima y otras ciudades del país. La desaparición de la cobertura vegetal deja al descubierto el estrato rocoso que queda expuesto a la erosión del 
agua y de los vientos, por lo que la regeneración natural es lenta y difícil debido a las bajas temperaturas que ocasionan disminución de la actividad microbiológica y en la tasa de descomposición orgánica.

En la región central de nuestro país la extracción de las turberas se realiza principalmente en lugares con fáciles vías de acceso a la ciudad capital; sin embargo, la sobreexplotación ha motivado la búsqueda de nuevos sitios de extracción en otras partes de nuestro territorio (o zonas aledañas). Así por ejemplo, los oconales de las lagunas Pomacocha y Habascocha en Junín se han constituido en uno de estos puntos de extracción.

El objetivo de este trabajo fue conocer la riqueza florística de la vegetación que circunda a estas lagunas, así como llamar la atención de los especialistas a fin de promover su manejo y conservación.

\section{Área de estudio}

Las lagunas Pomacocha y Habascocha cuyo origen glacial es similar al de la mayoría de lagunas altoandinas (Santayana, com. pers.)- están ubicadas en la cuenca alta del río Comas, distrito de Comas, en las alturas de la provincia de Concepción, departamento de Junín, a altitudes entre 4350 y 4550 m, cercanas al centro poblado Pampa-Corral. El territorio está situado entre las coordenadas $11^{\circ} 45^{\prime}-11^{\circ} 48^{\prime}$ 'S y $75^{\circ} 12^{\prime}-75^{\circ} 15^{\prime}$ 'W y tiene una extensión superficial estimada de $9 \mathrm{~km}^{2}$.

Fitogeográficamente forman parte de la Región Neotropical, Dominio AndinoPatagónico, Provincia Altoandina y Puneña según Cabrera y Willink (1980). En términos de Rivas-Martínez \& Tovar (referidos por Tovar, 1990) la zona de estudio se ubica dentro del Territorio Andino, en el Piso Bioclimático Altoandino o Puna inferior. De acuerdo a la ONERN (1976), las lagunas se sitúan en la zona de vida Páramo muy húmedo-subalpino Tropical (pmh-SaT), con una biotemperatura media anual de $6{ }^{\circ} \mathrm{C}$ y una media anual mínima de $3,8{ }^{\circ} \mathrm{C}$; el promedio máximo de precipitación total por año es de $1254,8 \mathrm{~mm}$ y el promedio mínimo de 584,2 $\mathrm{mm}$.

El relieve es casi llano con cerros de suave pendiente. El clima es frígido, por lo regular llueve todo el año, distinguiéndose dos estaciones: la hibernal y la estival.

A primera impresión la vegetación pareciera estar constituida básicamente por una abundante mezcla de gramíneas y otras diversas especies de hierbas; sin embargo, es posible reconocer cuatro tipos de comunidades en el área de estudio, a saber: el césped de puna, los oconales o turberas de Distichia, la comunidad de los roquedales y la comunidad de las hidrófitas.

\section{Material y métodos}

Las recolectas se llevaron a cabo dos veces por año, en los meses de marzo y abril en los años 1997 y 2004, y durante abril y junio en el 2000 y el 2001. La técnica de recolección fue la convencional (Smith, 1971). Las recolectas se realizaron de manera aleatoria y a diferentes altitudes en ambas lagunas, entre los 4350 y $4550 \mathrm{~m}$. La determinación de los géneros y las especies se realizó utilizando literatura pertinente -i.e. claves y descripciones publicadas en libros o revistas botánicaso con la ayuda de especialistas en algunas fa-

Tabla 1. Taxa registrados en las lagunas Pomacocha y Habascocha, Concepción, Junín, Perú.

\begin{tabular}{|c|c|c|c|c|c|c|}
\hline \multirow[t]{2}{*}{ Clase } & \multicolumn{2}{|c|}{ Familias } & \multicolumn{2}{|c|}{ Géneros } & \multicolumn{2}{|c|}{ Especies } \\
\hline & No & $\%$ & No & $\%$ & No & $\%$ \\
\hline $\begin{array}{l}\text { Dicotiledóneas } \\
\text { (Magnoliopsida) }\end{array}$ & 23 & 79 & 47 & 73 & 68 & 68 \\
\hline $\begin{array}{l}\text { Monocotiledóneas } \\
\text { (Liliopsida) }\end{array}$ & 6 & 21 & 17 & 27 & 32 & 32 \\
\hline TOTAL & 29 & 100 & 64 & 100 & 100 & 100 \\
\hline
\end{tabular}


Tabla 2. Familias con mayor número de géneros y especies.

\begin{tabular}{|c|c|c|c|c|}
\hline \multirow[t]{2}{*}{ Familias } & \multicolumn{2}{|c|}{ Géneros } & \multicolumn{2}{|c|}{ Especies } \\
\hline & $\mathbf{N}^{o}$ & $\%$ & $\mathbf{N}^{\circ}$ & $\%$ \\
\hline Poaceae & 10 & 15,6 & 25 & 25,0 \\
\hline Asteraceae & 15 & 23,4 & 24 & 24,0 \\
\hline Gentianaceae & 3 & 4,7 & 6 & 6,0 \\
\hline Campanulaceae & 1 & 1,6 & 5 & 5,0 \\
\hline Caryophyllaceae & 4 & 6,3 & 4 & 4,0 \\
\hline Scrophulariaceae & 4 & 6,3 & 4 & 4,0 \\
\hline Fabaceae & 1 & 1,6 & 3 & 3,0 \\
\hline Plantaginaceae & 1 & 1,6 & 3 & 3,0 \\
\hline Cyperaceae & 2 & 3,1 & 2 & 2,0 \\
\hline Juncaceae & 2 & 3,1 & 2 & 2,0 \\
\hline Ranunculaceae & 2 & 3,1 & 2 & 2,0 \\
\hline Solanaceae & 2 & 3,1 & 2 & 2,0 \\
\hline Malvaceae & $\begin{array}{l}1 \\
(48)\end{array}$ & $\begin{array}{l}1,6 \\
(75,0)\end{array}$ & $\begin{array}{l}2 \\
(84)\end{array}$ & $\begin{array}{l}2,0 \\
(84,0)\end{array}$ \\
\hline Otras familias & 16 & 25,0 & 16 & 16,0 \\
\hline TOTAL & 64 & 100 & 100 & 100 \\
\hline
\end{tabular}

milias; también se realizaron comparaciones con ejemplares depositados en el Herbario de la Universidad Nacional Agraria La Molina (MOL) y en el Herbario de la Universidad Nacional Mayor de San Marcos (USM). La colección completa se encuentra depositada en MOL.

Para el presente trabajo se ha utilizado el sistema de clasificación de las Angiospermas (Magnoliophyta) de Cronquist (1981).

\section{Resultados}

La flora fanerogámica de los alrededores de las lagunas Pomacocha y Habascocha, Concepción, Junín, está compuesta por 29 familias, 64 géneros y 100 especies.

Las dicotiledóneas (Magnoliopsida) representan el $79 \%$ de las familias, $73 \%$ de los géneros y $68 \%$ de las especies, mientras que las monocotiledóneas (Liliopsida) el 21, 27 y 32\% de las familias, géneros y especies respectivamente (Tabla 1).

Del total de especies, 94 son hierbas que representan el $94 \%$ y 6 son sufrútices que representan el $6 \%$. No se hallaron especies arbustivas ni arbóreas.

Las familias con mayor porcentaje de especies son: Poaceae con $25 \%$, Asteraceae con
$24 \%$, Gentianaceae con 6\%, Campanulaceae con 5\%, Caryophyllaceae con 4\%, Scrophulariaceae con 4\%, Fabaceae y Plantaginaceae ambas con 3\%, Cyperaceae, Juncaceae, Malvaceae, Ranunculaceae y Solanaceae todas con $2 \%$. Las familias restantes están representadas por 1 especie cada una, y constituyen el 16\% del total (Tabla 2).

Ambas, Poaceae y Asteraceae contribuyen con el 39\% de los géneros y el 49\% de las especies de la flora fanerogámica de las lagunas Pomacocha y Habascocha.

Los géneros con mayor número de especies son Calamagrostis (6), Dissanthelium (6), Lysipomia (5), Werneria (4) y Gentianella (4) (Tabla 3).

Por otro lado, la flora que se asocia a las lagunas se encuentra formando cuatro tipos de vegetación que, en buena medida, es posible diferenciar en función de las formas de vida predominantes y su clara relación con

Tabla 3. Géneros con mayor número de especies.

\begin{tabular}{ll}
\hline Género & Especie \\
\hline Calamagrostis & C. jamesonii \\
& C. minima \\
& C. pungens vel aff. \\
& C. rigescens \\
& C. spicigera vel aff. \\
& C. vicunarum \\
& D. breve \\
Dissanthelium & D. brevifolium vel aff. \\
& D. calycinum \\
& D. densum vel aff. \\
& D. expansum vel aff. \\
& D. macusaniense \\
& L. sp 1 \\
Lysipomia & L. sp 2 \\
& L. sp 3 \\
& L. sp 4 \\
& L. sp 5 \\
W. caespitosa \\
W. nubigena \\
Werneria & W. pectinata \\
& W. pygmaea \\
& G. calcarea \\
& G. chrysosphaera \\
& G. limoselloides \\
& G. nitida \\
&
\end{tabular}


ambientes determinados. Así, acorde con la terminología de Weberbauer (1945) es posible distinguir:
a. Césped de puna
b. Comunidad de roquedales
c. Oconales o turbera de Distichia
d. Comunidad de hidrófitas

\section{a. Césped de puna}

Se caracteriza por una vegetación baja de hierbas rizomatosas que ocupan terrenos más o menos planos, con suelos poco pedregosos y medianamente húmedos. Predominan las especies graminiformes que se asocian en matas, otras especies forman cojines planos o convexos, varias otras poseen hojas en roseta. Aunque poco conspicuos, también suelen crecer sufrútices postrados. Los elementos más comunes son Aciachne pulvinata, Aciachne acicularis "paco-champa»y Calamagrostis vicunarum "crespillo», asociadas a otras gramíneas como Agrostis breviculmis, Calamagrostis minima, Dissanthelium calycinum, Dissanthelium macusaniense, Festuca peruviana, junto a ellas destacan las asteráceas: Werneria nubigena, Werneria pygmaea, Baccharis caespitosa, Senecio repens, Gamochaeta oreophila, Cuatrecasasiella isernii, además de las gentianáceas: Gentianella chrysosphaera, Gentiana sedifolia; malváceas como Nototriche pinnata, geraniáceas como Geranium pavonianum y rosáceas como Alchemilla pinnata.

\section{b. Comunidad de roquedales}

En varios lugares de las colinas que rodean a las lagunas emergen enormes afloramientos rocosos cuya superficie contiene un suelo poco profundo, producto de la erosión, además del detrito vegetal acumulado por varios años. En la base de estas rocas y protegidas entre las grietas viven todo el año algunas hierbas y sufrútices favorecidos por la humedad.

Entre las herbáceas encontramos a Oreomyrrhis andicola, Urtica flabellata,
Lupinus andinus, y entre las de hábito sufruticoso a Lupinus ananeanus, Salpichroa glandulosa y Muehlenbeckia volcanica.

\section{c. Oconales o turbera de Distichia}

Los oconales son lugares donde el suelo está permanentemente inundado formando vegas o ciénagas; los cursos de agua provienen del deshielo de los glaciares. Son sitios «muy estimados por los ganaderos porque constituyen bebederos naturales» (Cerrate, 1979). En estos lugares se establece una flora herbácea, donde el componente más importante es la juncácea Distichia muscoides, que forma grandes cojines planos o convexos muy compactos.

En asociación con $D$. muscoides crecen: Plantago rigida «champa estrella», además de frecuentes especies pigmeas o arrosetadas de dicotiledóneas como varias campanuláceas del género Lysipomia, escrofulariáceas como Ourisia muscosa, asteráceas como Cuatrecasasiella isernii y Oritrophium limnophilum, pero también poáceas como Calamagrostis rigescens, Poa aequigluma, entre otras.

\section{d. Comunidad de hidrófitas}

Es característica de las aguas estancadas o de corriente lenta. En la puna, las lagunas se forman por el deshielo de los glaciares; además, el agua proveniente de pequeñas corrientes, que discurre entre los oconales y el césped de puna, o el de las lluvias se deposita en lugares poco profundos para formar pozos o charcos, algunos de ellos efímeros.

La vegetación de hidrófitas se puede subdividir en sumergidas y anfibias.

\section{Comunidad de hierbas sumergidas}

Las especies más comunes son Myriophyllum quitense vel aff. y Elodea potamogeton, cuyas ramas se mezclan profusamente.

\section{Comunidad de anfibias}

Estas plantas se establecen en ambientes 
acuáticos, pero cuando ocurre la desecación por falta de lluvia o inundación, pueden sobrevivir temporalmente gracias a adaptaciones vegetativas. Entre ellas están Ranunculus limoselloides, Mimulus glabratus y Lysipomia sp.

\section{Discusión}

El estado actual del conocimiento de los humedales altoandinos del Perú y sus características ecológicas resulta aún insuficiente (León, 1993; Castro \& Pulido, 1996); sin embargo, este estudio muestra que existe una importante diversidad florística en la puna de Concepción (Junín), en los territorios circundantes a las lagunas de Pomacocha y Habascocha.

La flora fanerogámica de la puna de Concepción comprende 29 familias, 64 géneros y 100 especies; el 79\% de la flora está compuesta por familias de dicotiledóneas y el $21 \%$ por monocotiledóneas.

Del total de las 100 especies que se reportan, 18 de ellas constituyen endemismos del Perú, 21 no están citadas por Brako y Zarucchi (1993) para el departamento de Junín; además, 4 de las mismas pertenecen a géneros no registrados para dicho departamento (Jalcophila, Montia, Oreobolopsis y Plagiobothrys).

La proporción relativa entre dicotiledóneas y monocotiledóneas, así como la predominancia de las familias Asteraceae y Poaceae, es similar a la que se presenta en otras localidades altoandinas en el Perú y concuerda con lo señalado por Gentry (en Brako \& Zarucchi, 1993) cuando afirma que las Asteraceae y Poaceae son las familias dominantes y más ricas específicamente por encima del límite de los bosques. Estas mismas relaciones han sido halladas por Sánchez (1996) para la jalca del Perú, Jørgensen \& Ulloa Ulloa(1994) y Luteyn (1996) para el páramo y por Cabrera (1957) para la puna de Argentina.

En la familia Poaceae, la subfamilia con mayor número de especies (23) es Pooideae (tribus Poeae y Stipeae).

En la familia Asteraceae, las tribus mejor representadas y con mayor número de especies son Gnaphalieae, Senecioneae y Astereae.

Es interesante apuntar que las familias Poaceae y Asteraceae reúnen juntas 25 géneros $(39 \%)$ y 49 especies (49\%), lo que implica que la diversidad en la flora fanerogámica estudiada está dispersa en 27 familias que contienen entre 1 a 6 especies cada una, lo que en términos porcentuales representa el $51 \%$.

Las hierbas constituyen el $94 \%$ de las especies, predominan las formas enanas, arrosetadas, acaules y las pulviniformes (Aciachne pulvinata, Aciachne acicularis, Plantago rigida), unas cuantas especies son acuáticas sumergidas como Elodea potamogeton y Myriophyllum quitense vel aff. o anfibias como Ranunculus limoselloides y Mimulus glabratus. Los subarbustos de los géneros Baccharis, Lupinus, Muehlenbeckia, Salpichroa y Senecio representan solo el 6\%. Esta evidente predominancia del hábito herbáceo por encima de los $4000 \mathrm{msm}$. se ajusta a lo reportado por Weberbauer (1945), Cabrera (1957) y Jørgensen \& Ulloa Ulloa(1994).

De acuerdo a nuestras primeras observaciones son cuatro las comunidades vegetales, ya mencionadas, asociadas a los humedales de la puna de Concepción que se pueden distinguir; no obstante, debemos aclarar que el principal objetivo de este estudio fue documentar la diversidad y composición florística de este territorio; no hubo tiempo de llevar a cabo estudios detallados de zonas de vegetación. Cabrera (1957), respecto de la vegetación altoandina, refiere que las relaciones entre especies vegetales dominantes y especies secundarias son generalmente poco claras, asimismo añade que las relaciones sucesionales entre las diferentes comunidades no son evidentes «ya que no existe una evolución del 
suelo paralela a la de la vegetación ni tienen los vegetales influencia aparente sobre el suelo. Sólo en algunas comunidades higrófilas se aprecia la formación de suelos de turbera».

\section{Relación de la flora de la puna de Con- cepción con la flora de otras localidades altoandinas}

A pesar de que el área estudiada es pequeña, la riqueza florística de la puna de Concepción (Junín) es proporcionalmente alta y su composición ofrece semejanzas manifiestas con la de otras localidades altoandinas (Tabla 4).

Así, al relacionar los resultados obtenidos en este estudio con la flora de la puna del Parque Nacional Huascarán (Smith, 1988) se encuentra que en ésta, las dicotiledóneas consti- tuyen el $80 \%$ y las monocotiledóneas el 20\%. Las floras de ambas localidades, Concepción y el P. N. Huascarán, tienen en común 27 familias, 58 géneros y 57 especies, en ambas predominan las familias Asteraceae y Poaceae con $49 \%$ y $37 \%$ de riqueza específica, respectivamente.

La puna de la Reserva Nacional de Pampa Galeras (Tovar, 1973) muestra también significativas coincidencias, aquí el $81 \%$ de la flora fanerogámica se compone de dicotiledóneas y el 19\% de monocotiledóneas. La puna de Concepción comparte con la R. N. de Pampa Galeras, 21 familias, 31 géneros y 26 especies; de igual manera, las familias Poaceae y Asteraceae resultan las más diversas, reuniendo el $57 \%$ de las especies.

Tabla 4. Número de géneros y especies compartidas de las familias comunes a la puna de Concepción (C), el Parque Nacional Huascarán (H), la Reserva Nacional de Pampa Galeras (G) (Tovar, 1973) y el pajonal del Parque Nacional Río Abiseo (A) (Young \& León, 1990).

\begin{tabular}{|c|c|c|c|c|c|c|c|}
\hline \multirow[t]{2}{*}{ Familia } & \multicolumn{4}{|c|}{$\mathbf{N}^{\circ}$ Géneros/ $\mathbf{N}^{\circ}$ Especies } & \multicolumn{3}{|c|}{$\begin{array}{c}\mathbf{N}^{0} \text { Géneros/ } \mathbf{N}^{\circ} \text { Especies } \\
\text { Compartidos }\end{array}$} \\
\hline & C & $\mathbf{H}$ & G & A & $\mathrm{C} / \mathrm{H}$ & $\mathrm{C} / \mathrm{G}$ & C/A \\
\hline Apiaceae & $1 / 1$ & $7 / 12$ & $3 / 3$ & $5 / 6$ & $1 / 1$ & -- & $1 / 1$ \\
\hline Asteraceae & $15 / 24$ & $59 / 164$ & $15 / 26$ & $21 / 41$ & $14 / 18$ & $5 / 4$ & $5 / 2$ \\
\hline Boraginaceae & $1 / 1$ & $3 / 3$ & $1 / 1$ & - & $1 / 0$ & $1 / 0$ & - - \\
\hline Campanulaceae & $1 / 5$ & $5 / 9$ & -- & $2 / 3$ & $1 / 0$ & -- & $1 / 0$ \\
\hline Caryophyllaceae & $4 / 4$ & $10 / 24$ & $3 / 4$ & $3 / 3$ & $4 / 2$ & $1 / 0$ & $1 / 0$ \\
\hline Cyperaceae & $2 / 2$ & $8 / 20$ & $3 / 3$ & $6 / 14$ & $1 / 0$ & $1 / 0$ & $1 / 0$ \\
\hline Fabaceae & $1 / 3$ & $9 / 27$ & $3 / 8$ & $3 / 3$ & $1 / 1$ & $1 / 2$ & $1 / 0$ \\
\hline Gentianaceae & $3 / 6$ & $3 / 9$ & $1 / 3$ & $3 / 5$ & $3 / 1$ & $1 / 0$ & $3 / 0$ \\
\hline Geraniaceae & $1 / 1$ & $2 / 4$ & $1 / 1$ & $1 / 2$ & $1 / 0$ & $1 / 0$ & $1 / 0$ \\
\hline Haloragaceae & $1 / 1$ & $1 / 1$ & -- & -- & $1 / 1$ & -- & - \\
\hline Hydrocharitaceae & $1 / 1$ & -- & $1 / 1$ & -- & $1 / 1$ & $1 / 1$ & -- \\
\hline Iridaceae & $1 / 1$ & $3 / 9$ & $1 / 1$ & $1 / 2$ & $1 / 0$ & -- & -- \\
\hline Juncaceae & $2 / 2$ & $3 / 12$ & $2 / 3$ & $2 / 4$ & $2 / 2$ & $2 / 2$ & $1 / 1$ \\
\hline Lamiaceae & $1 / 1$ & $5 / 8$ & -- & $1 / 1$ & $1 / 1$ & -- & -- \\
\hline Malvaceae & $1 / 2$ & $3 / 11$ & $4 / 5$ & - & $1 / 1$ & $1 / 0$ & -- \\
\hline Orchidaceae & $1 / 1$ & $11 / 20$ & -- & $5 / 6$ & $1 / 1$ & -- & -- \\
\hline Oxalidaceae & $1 / 1$ & $2 / 4$ & -- & -- & $1 / 0$ & -- & -- \\
\hline Plantaginaceae & $1 / 3$ & $1 / 5$ & $2 / 2$ & $1 / 2$ & $1 / 3$ & $1 / 1$ & $1 / 0$ \\
\hline Polygonaceae & $1 / 1$ & $2 / 4$ & -- & $2 / 3$ & $1 / 1$ & -- & $1 / 1$ \\
\hline Poaceae & $10 / 25$ & $32 / 97$ & $13 / 55$ & $9 / 15$ & $9 / 13$ & $8 / 12$ & $5 / 1$ \\
\hline Ranunculaceae & $2 / 2$ & $3 / 8$ & $1 / 2$ & $3 / 4$ & $1 / 1$ & $1 / 1$ & $1 / 1$ \\
\hline Rosaceae & $1 / 1$ & $9 / 18$ & $3 / 4$ & $3 / 4$ & $1 / 1$ & $1 / 1$ & $1 / 0$ \\
\hline Rubiaceae & $1 / 1$ & $2 / 5$ & $1 / 1$ & $3 / 3$ & $1 / 1$ & $1 / 0$ & $1 / 0$ \\
\hline Scrophulariaceae & $4 / 4$ & $12 / 26$ & $3 / 3$ & $4 / 5$ & $4 / 4$ & $2 / 0$ & $1 / 0$ \\
\hline Solanaceae & $1 / 1$ & $8 / 26$ & $2 / 2$ & -- & $2 / 1$ & $1 / 1$ & -- \\
\hline Urticaceae & $1 / 1$ & $2 / 4$ & $1 / 1$ & -- & $1 / 1$ & $1 / 1$ & -- \\
\hline Violaceae & $1 / 1$ & $1 / 2$ & -- & $1 / 2$ & $1 / 1$ & -- & $1 / 0$ \\
\hline
\end{tabular}


Por otro lado, la puna del Parque Nacional del Río Abiseo (Young \& León, 1990) alberga una flora donde el $66 \%$ está constituida por dicotiledóneas y el $34 \%$ por monocotiledóneas, las familias de importancia son también Asteraceae y Poaceae, sumando juntas el 34\% del total de las especies (Cano \& Young, 1994); encontramos 20 familias, 27 géneros y sólo 7 especies comunes a la flora de Concepción. Esta última disimilitud bien podría deberse a que el sector comprendido entre 3200 - 4200 $\mathrm{m}$ del P. N. del Río Abiseo corresponde a un «páramo» (en términos de Young \& León, 1990), conformado básicamente por pajonales, contrastando con la puna de Concepción, donde parecieran predominar el césped de puna y los oconales. No obstante cabe indicar que, pese a estas aparentes diferencias, nuestro estudio revela que la vegetación de la puna de Concepción muestra presuntos vínculos comparativamente más cercanos con territorios fitogeográficos altoandinos tropicales como el páramo o la jalca que con la puna subtropical que se extiende al sur del departamento de Junín. Sánchez (1996) señala que la jalca, en tanto zona transicional, posee numerosas especies comunes al páramo y la puna; pero asimismo, afirma la existencia de géneros y especies, algunos endémicos, que caracterizan tal territorio desde el punto de vista florístico. A este respecto, resulta sorpresivo nuestro hallazgo de una especie probablemente nueva de Jalcophila, género hasta hoy confinado a los páramos colombiano-ecuatorianos y endémico, en Perú, de la jalca de Santiago de Chuco en el departamento de La Libertad (Dillon \& Sagástegui, 1991) -omitido en el recuento de Sánchez (1996)-. La presencia de los géneros Oritrophium (Asteraceae), Lysipomia (Campanulaceae), Luzula (Juncaceae), o de especies tales como Cuatrecasasiella isernii o Plantago rigida de distribución característicamente paramuna es sugestiva y pareciera reforzar nuestra presunción.

Finalmente, Tovar (1990) hace referencia a las especies más comunes que se encuentran en las lagunas altoandinas de la cuenca del
Mantaro, coincidiendo en forma general con los resultados obtenidos en el presente estudio.

Es importante mencionar que los inventarios de las floras de la R. N. de Pampa Galeras (Ayacucho) y P. N. del Río Abiseo (La Libertad-San Martín) no reflejan la diversidad de sus especies en su totalidad ya que no han sido totalmente estudiadas.

\section{Implicancias para la conservación}

Este estudio muestra que existe una importante riqueza florística cuyo conocimiento debe servir de base para su manejo y conservación, por ser zona de pastoreo y extracción de la turbera de Distichia para su utilización en viveros o como combustible. La depredación del «musgo» Distichia muscoides y de otras especies que conviven con ésta es uno de los problemas que afectan a los oconales. Ya desde el año 1996, se observó la depredación de este «musgo» en 2000 hectáreas de las 4000 hectáreas que existen en las cumbres andinas de la cuenca del río Rímac. La extracción en los oconales «deja un panorama desolador constituido por enormes hoyos negros que a lo lejos dan la impresión de ser campos calcinados» (Necochea, 1998).

Los especialistas han señalado que la depredación de $D$. muscoides ocasiona daños irreversibles, pues esta especie se reproduce en un período de 80-100 años (Necochea, 1998).

La vegetación altoandina es una de las menos conocida y menos protegida. Nuestro estudio ha demostrado que existe una riqueza florística susceptible de ser manejada racionalmente; más aun, que este ecosistema de alta montaña está sometido a impacto humano, debido a su cercanía a la carretera de penetración a la ceja de montaña.

Sugerimos la tarea de iniciar una educación ambiental dirigida a las comunidades locales, para promover un conocimiento mayor de su entorno y el uso racional de sus recursos naturales. 


\section{Agradecimientos}

Los autores agradecen a la Dra. Magda Chanco por su ayuda en la determinación de las especies de Malvaceae, al Biólogo Asunción Cano por permitirnos revisar la colección depositada en USM. Nuestro reconocimiento a la Lic. Isabel Murillo y al Ing. Toribio Santayana por los datos proporcionados sobre las lagunas. A los señores Carlos y Héctor Medina por su valioso apoyo logístico en el trabajo de campo y a la Sra. Elida Galarza de Medina por todas las facilidades en el alojamiento.

\section{Literatura citada}

Brako, L. \& J. L. Zarucchi. 1993. Catalogue of the Flowering Plants and Gymnosperms of Peru. Monogr. Syst. Bot. Missouri Bot. Gard. 45:11286.

Cabrera, A. L. 1957. La vegetación de la Puna Argentina. INTA, Rev. Invest. Agric. 11(4): 317-412.

Cabrera, A. L. 1968. Ecología vegetal de la puna. Colloq. Geographicum 9: 91-116.

Cabrera, A. L. \& A. Willink. 1980. Biogeografía de América Latina. Serie Biol., Monogr. 13: 1-122. Dpto. Asuntos Científicos y Tecnológicos, O.E.A. Washington, D.C.

Castro, G. \& V. Pulido. 1996. Humedales del Perú. Pp. 103-104. En: L. O. Rodríguez (ed.), Diversidad Biológica del Perú: Zonas Prioritarias para su Conservación. Proyecto FANPE GTZ INRENA.

Cerrate, E. 1979. Vegetación del Valle de Chiquián (Departamento de Ancash). Edit. Los Pinos, Lima.

Cronquist, A. 1981. An Integrated System of Classification of Flowering Plants. Columbia Univ. Press, New York.

Dillon, M. O. \& A. Sagástegui. 1991. Sinopsis de los géneros de Gnaphaliinae (Asteraceae - Inuleae) de Sudamérica. Arnaldoa 1(2): 5-91.

Gentry, A. H. 1993. Overview of the Peruvian flora. In: L. Brako \& J. L. Zarucchi (eds.), Catalogue of the Flowering Plants and Gymnosperms of Peru. Monogr. Syst. Bot. Missouri Bot. Gard. 45: xxix-xl.

Gómez, J. 1966. Notas sobre la vegetación del valle de Marca. Biota 6(47): 93-123.

Jørgensen, P. M. \& C. Ulloa Ulloa. 1994. Seed Plants of the High Andes of Ecuador - A checklist. AAU Reports 34: 1-443.
León, B. 1993. Catálogo anotado de las fanerógamas acuáticas del Perú. En: F. Kahn, B. León \& K. R. Young (eds.), Las Plantas Vasculares en las Aguas Continentales del Perú. Travaux de l'Institut Francais d'Etudes Andines Tomo 75. IFEA (Institut Francais d'Etudes Andines), Lima. Pp. 11-128.

Luteyn, J. L. 1996. Fitodiversidad y conservación del páramo. En: Anales del Simposio Estrategias para Bioconservación en el Norte del Perú. Arnaldoa Ed. Esp. 4(2): 15-24.

Necochea, C. 1998. Daño en la cumbre. Diario El Comercio, Sec. Ecología, pp. D1. Lima, Perú.

Oficina Nacional de Evaluación de Recursos Naturales (ONERN). 1976. Mapa Ecológico del Perú. Guía Explicativa y Mapa. Lima.

Sánchez V., I. 1996. Aspectos florísticos de la jalca y alternativas de manejo sustentable. En: Anales del Simposio Estrategias para Bioconservación en el Norte del Perú. Arnaldoa Ed. Esp. 4(2): 25-62.

Smith, C. E. 1971. Preparing herbarium specimens of vascular plants. Agric. Information Bull. 348: $1-29$.

Smith, D. N. 1988. Flora and Vegetation of the Huascaran National Park, Ancash, Peru, with Preliminary Taxonomic Studies for a Manual of the Flora. Unpublished Ph.D. dissertation, Iowa State Univ., Ames, IA.

Tapia, M. \& J. Flores. 1984. Pastoreo y Pastizales de los Andes del Sur del Perú. INIPA. Edit. Adolfo Artela. Lima, Perú.

Tovar, O. 1973. Comunidades vegetales de la Reserva Nacional de Vicuñas de Pampa Galeras, Ayacucho, Perú. Publ. Mus. Hist. Nat. Javier Prado, Serie B, Bot. 27: 1-32.

Tovar, O. 1990. Tipos de Vegetación, Diversidad Florística y Estado de Conservación de la Cuenca del Mantaro. Centro de Datos para la Conservación. Univ. Nac. Agr. La Molina. Lima, Perú.

Weberbauer, A. 1945. El Mundo Vegetal de los Andes Peruanos. Estac. Exper. Agric. La Molina. Edit. Lumen. Lima, Perú.

Young, K. R. \& B. León. 1990. Catálogo de las plantas de la zona alta del Parque Nacional Río Abiseo, Perú. Publ. Mus. Hist. Nat. UNMSM (B) 34: $1-37$.

Young, K. R. \& A. Cano. 1994. Aporte florístico de la puna del Parque Nacional del Manu, Perú. Bol. Lima 16: 381-393. 
Anexo 1. Familias y especies de la flora fanerogámica de las lagunas Pomacocha y Habascocha, Concepción, Junín (forma de crecimiento: $\mathrm{H}$ = Hierba, $\mathrm{S}=$ Subarbusto).

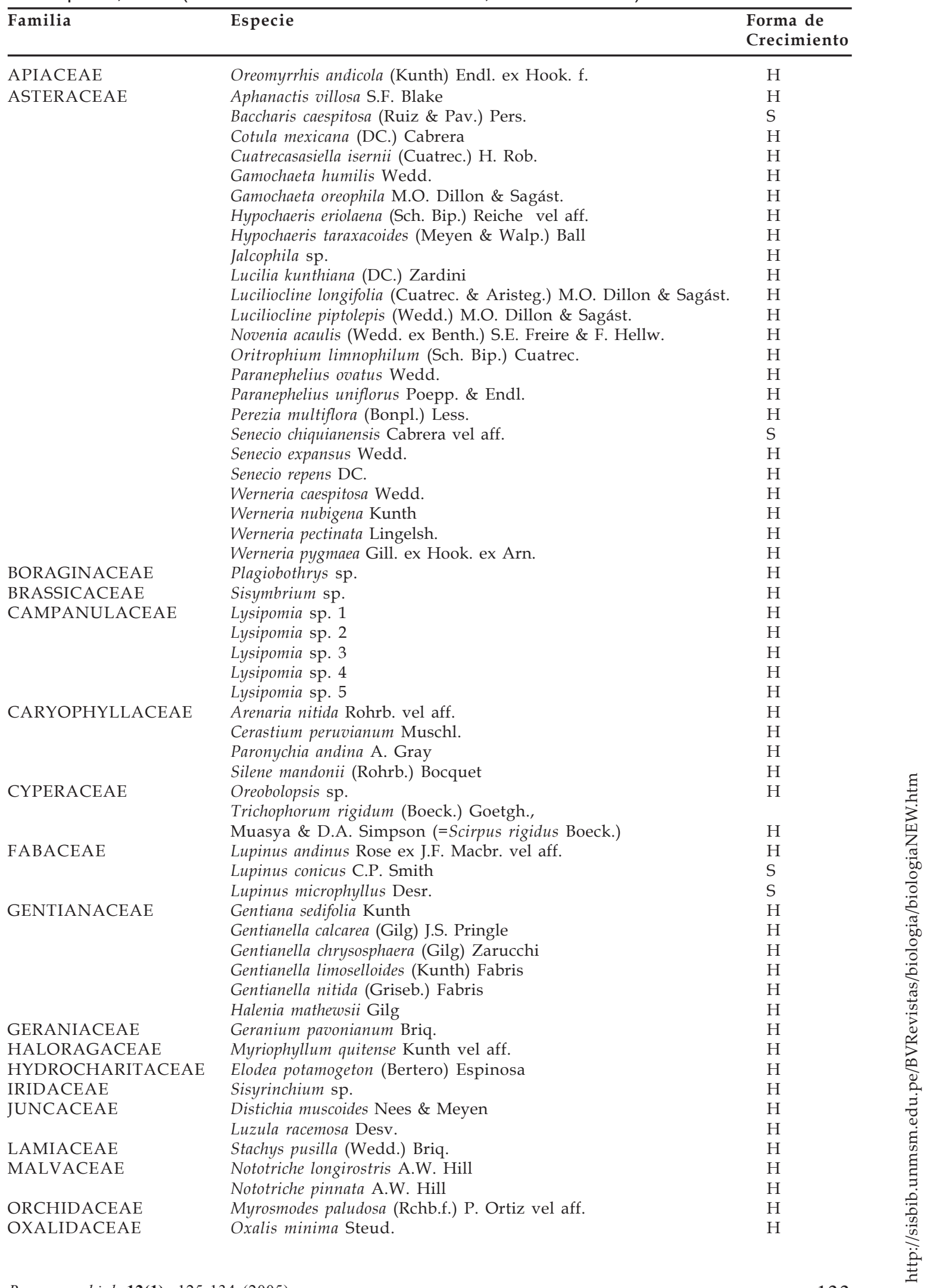


$\begin{array}{ll}\text { PLANTAGINACEAE } \quad \text { Plantago lamprophylla Pilg. } & \mathrm{H}\end{array}$

Plantago rigida Kunth $\quad \mathrm{H}$

$\begin{array}{lll} & \text { Plantago tubulosa Decne. } & \mathrm{H} \\ \text { POACEAE } & \text { Aciachne acicularis Laegaard } & \mathrm{H}\end{array}$

$\begin{array}{ll}\text { Aciachne pulvinata Benth. } & \mathrm{H} \\ \text { Agrostis breviculmis Hitchc. } & \mathrm{H}\end{array}$

$\begin{array}{ll}\text { Agrostis breviculmis Hitchc. } & \mathrm{H} \\ \text { Anatherostipa hans-meyeri (Pilg.) Peñailillo } & \mathrm{H}\end{array}$

Bromus lanatus Kunth $\quad \mathrm{H}$

Calamagrostis jamesonii Steud. $\quad \mathrm{H}$

Calamagrostis minima (Pilg.) Tovar $\quad \mathrm{H}$

Calamagrostis pungens Tovar vel aff. $\mathrm{H}$

Calamagrostis rigescens (J. Presl) Scribn. $\quad \mathrm{H}$

Calamagrostis spicigera (J. Presl) Steud. vel aff. H

Calamagrostis vicunarum (Wedd.) Pilg. $\quad \mathrm{H}$

Dissanthelium breve Swallen \& Tovar $\quad \mathrm{H}$

Dissanthelium brevifolium Swallen \& Tovar vel aff. $\quad \mathrm{H}$

Dissanthelium calycinum (J. Presl) Hitchc. H

Dissanthelium densum Swallen \& Tovar vel aff. H

Dissanthelium expansum Swallen \& Tovar vel aff. H

Dissanthelium macusaniense (E.H.L. Krause) R.C. Foster \& L.B. Sm. H

Festuca casapaltensis Ball $\quad \mathrm{H}$

Festuca peruviana Infantes $\quad \mathrm{H}$

Hordeum muticum J. Presl H

Muhlenbergia ligularis (Hack.) Hitchc. $\quad \mathrm{H}$

Muhlenbergia peruviana (P. Beauv.) Steud. $\mathrm{H}$

Poa aequigluma Tovar $\quad \mathrm{H}$

Poa annua L. $\quad \mathrm{H}$

Poa gymnantha Pilg. vel aff. $\quad \mathrm{H}$

POLYGONACEAE $\quad$ Muehlenbeckia volcanica (Benth.) Endl. $\quad$ S

PORTULACACEAE Montia fontana L. $\quad$ H

RANUNCULACEAE $\quad$ Oreithales integrifolia (DC.) Schltdl. H

ROSACEAE Ranunculus limoselloides Turcz.

RUBIACEAE $\quad$ Galium hypocarpium Endl. ex Griseb. $\quad$ H

SCROPHULARIACEAE Bartsia diffusa Benth.

Castilleja pumila (Benth.) Wedd. $\quad \mathrm{H}$

Mimulus glabratus Kunth $\quad \mathrm{H}$

Ourisia muscosa Benth. $\mathrm{H}$

SOLANACEAE Salpichroa glandulosa (Hook.) Miers $\quad$ S

Solanum acaule Bitter $\quad \mathrm{H}$

$\begin{array}{lll}\text { URTICACEAE } & \text { Urtica flabellata Kunth } & \mathrm{H} \\ \text { VIOLACEAE } & \text { Viola pygmaea Juss. ex Poir. } & \mathrm{H}\end{array}$ 